\title{
Le projet ANR SOLCYP+ pour améliorer le dimensionnement des monopieux utilisés comme fondations d'éoliennes marines
}

\author{
Jean-Claude Dupla ${ }^{1 \star \star}$, Elisabeth Palix ${ }^{2}$, Guillaume Damblans ${ }^{3}$, Alain Puech ${ }^{4}$, Mathieu Blanc ${ }^{5}$, \\ Christophe Dano ${ }^{6}$, Hussein Mroueh ${ }^{7}$, Marc Kham ${ }^{8}$, Yiorgos Perikleous ${ }^{9}$, Yves Marin ${ }^{10}$ et Sebastien \\ Burlon $^{11}$
}

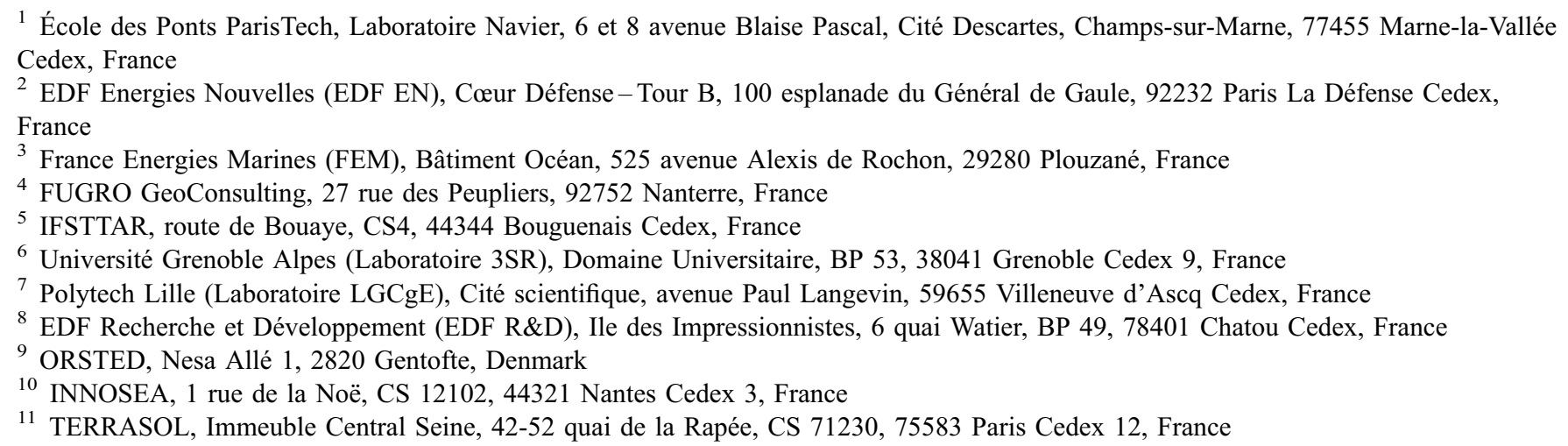

Résumé - La plupart des éoliennes offshore sont fondées sur monopieux. L'augmentation constante de la capacité des turbines et l'insuffisance des procédures évoquées dans les documents normatifs, notamment en ce qui concerne la prise en compte des chargements cycliques, posent des défis pour leur dimensionnement. Dans le cas des sites français, la présence de sols et roches carbonatés, dont le comportement est encore mal connu aujourd'hui, rend la tâche encore plus complexe. Le projet SOLCYP+, lancé en 2017 pour une durée de 3 ans, doit permettre de disposer de nouvelles méthodologies fiables et robustes pour le dimensionnement des monopieux de grand diamètre y compris dans le contexte géotechnique du plateau continental français. Cet article présente une description détaillée du projet ANR SOLCYP+. On aborde, tout d'abord, le contexte, la problématique et les objectifs du projet puis, dans un second temps, on décrit plus en détail les quatre principales tâches scientifiques.

Mots clés : offshore / éoliennes / monopieux / cyclique / dimensionnement

\begin{abstract}
The ANR SOLCYP+ project for improving the design of monopiles used as offshore wind turbines foundations. A large majority of offshore wind turbines are founded on monopiles. The constantly increasing capacity of the turbines and the limitations of the procedures described in the design codes, namely with regards to the consideration of cyclic loadings, result in challenging design issues. On the future French wind farm sites, the presence of carbonate soils and rocks has been identified. Their behavior being poorly understood, the complexity of the design is increased. The SOLCYP+ project, started in 2017 for a 3yearperiod, is intended to develop new reliable and robust procedures for designing large diameter monopiles, including in the French geotechnical context. This paper presents an overview of the ANR SOLCYP+ project. First, the context, the problematic and the objectives of the project are discussed and then the four main scientific work packages are described in detail.
\end{abstract}

Keywords: offshore / wind turbines / monopiles / cyclic / design

\footnotetext{
$\bar{\star}$ Auteur de correspondance : jean-claude.dupla@enpc.fr
} 


\section{Introduction}

Depuis trois décennies, le secteur de l'éolien en mer a crû de manière exponentielle, en particulier en Europe du Nord (mer du Nord, mer Baltique et mer d'Irlande) où sont regroupées environ $90 \%$ des installations. Cette source d'énergie devient de plus en plus intéressante avec des coûts de production qui ne cessent de baisser.

En ce qui concerne la France, son parc éolien est à ce jour uniquement terrestre mais elle a pour objectif, dans son projet de programmation pluriannuelle de l'énergie (PPE) de 2019 (Source: Ministère de la Transition écologique et solidaire, 2019), d'atteindre $2400 \mathrm{MW}$ d'énergie renouvelable d'origine marine à l'horizon 2023, et $6000 \mathrm{MW}$ à l'horizon 2028. Il s'agira essentiellement d'éoliennes en mer posées sur le fond.

Une grande majorité ( $~ 81 \%)$ des éoliennes offshore est actuellement fondée sur des monopieux (Negro et al., 2017) de grand diamètre (6 à $8 \mathrm{~m}$ ), pour des puissances comprises entre 4 et $8 \mathrm{MW}$. On envisage, dans un proche avenir, des éoliennes de plus forte puissance, d'environ $10 \mathrm{MW}$, qui nécessiteront des diamètres de monopieux plus importants (Velarde et Bachynski, 2017). Les fondations peuvent représenter jusqu'à $25 \%$ des coûts de l'investissement total (Fingersh et al., 2006) d'un parc éolien en mer (50 à 200 éoliennes). L'optimisation et la fiabilisation de leur dimensionnement est un problème majeur des acteurs du secteur. Les documents normatifs applicables au dimensionnement de monopieux font largement référence à des méthodologies définies pour l'industrie pétrolière offshore, développées pour les pieux flexibles (élancements importants) et qui ne sont pas adaptées au cas des monopieux de grand diamètre qui ont un comportement de type rigide (élancements faibles).

De plus, la plupart des nouveaux sites identifiés pour les appels d'offres gouvernementaux français sont composés de sols et roches carbonatés dont les propriétés géotechniques sont mal connues et le comportement incertain.

Le projet ANR SOLCYP+, piloté par FEM (France Energie Marine), a été lancé en février 2017 pour une durée de 3 ans. Il a pour ambition de répondre aux besoins fondamentaux du secteur de l'éolien posé : améliorer les méthodes de dimensionnement des monopieux et approfondir la connaissance du comportement des sols et roches carbonatés. Ce projet regroupe les acteurs industriels et académiques suivants: EDF-Energies Nouvelles, EDF Recherche \& Développement, FUGRO, INNOSEA, IFSTTAR (Département GERS), ORSTED, École des Ponts ParisTech, Polytech Lille (LGCgE), Université Grenoble Alpes (Laboratoire 3SR),

On détaille ci-après le contexte, la problématique et les objectifs du projet SOLCYP+, puis on décrit les quatre lots scientifiques du projet (lot 2 à lot 5).

\section{Problématique du dimensionnement des monopieux}

Le monopieu est le type de fondation majoritairement utilisé en Europe du Nord, car jugé économiquement le plus attractif (Fig. 1). Il devrait le rester dans les profondeurs d'eau représentatives des sites offshore français (la cinquantaine de mètres), même en tenant compte de l'augmentation prévisible de la taille des turbines. Les monopieux sont des tubes

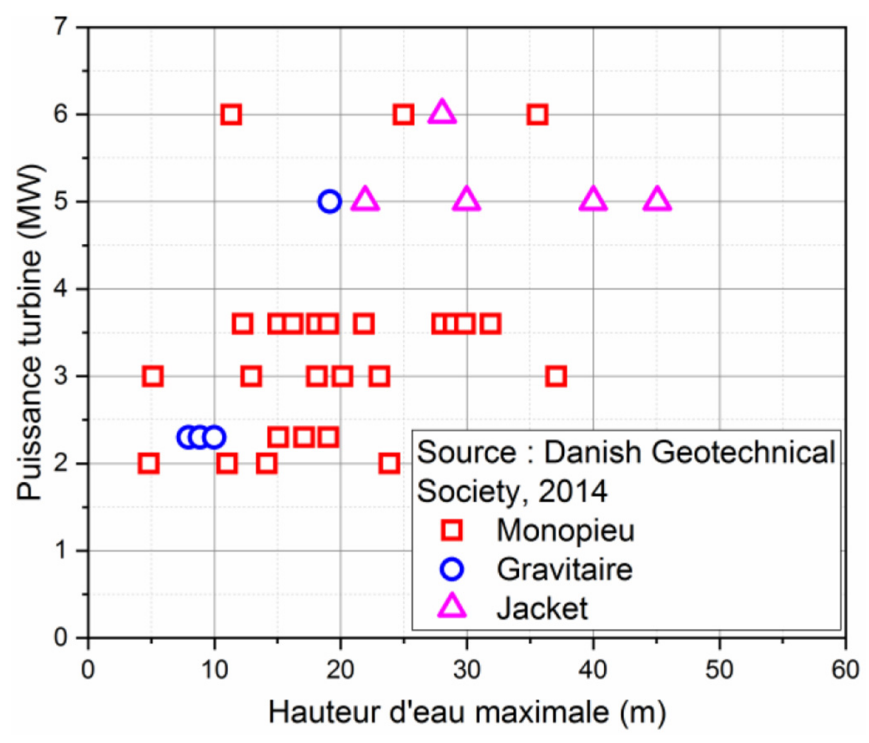

Fig. 1. Les fondations des éoliennes marines en 2014 (source : Danish Geotechnical Society, 2014).

Fig. 1. Wind turbines foundations in 2014 (source: Danish Geotechnical Society, 2014).

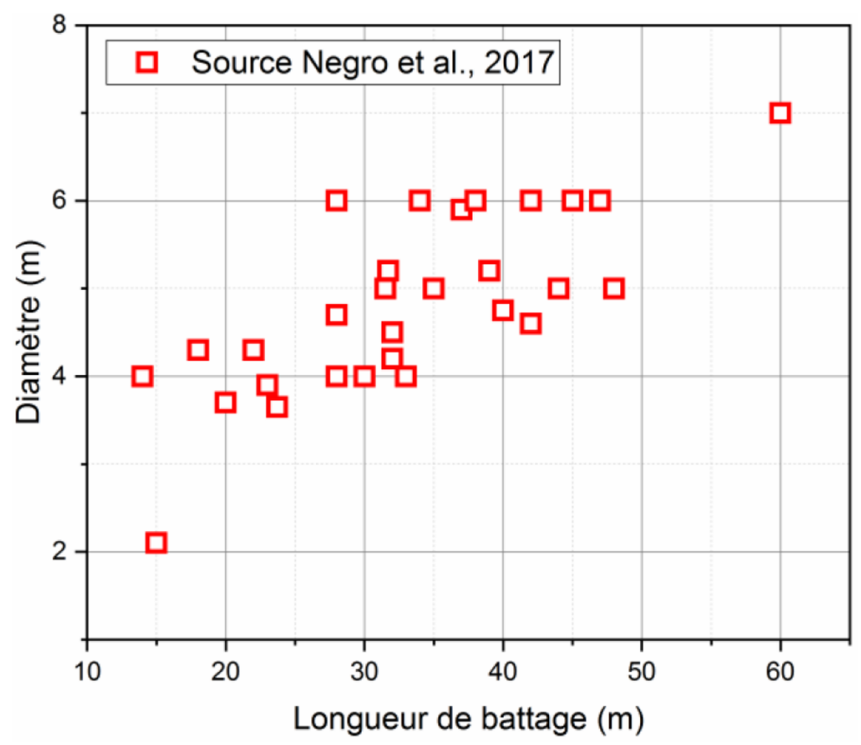

Fig. 2. Diamètre en fonction de la longueur de battage des monopieux des éoliennes marines d'après Negro et al., 2017.

Fig. 2. Diameter function of the driving length of offshore wind turbines monopiles from Negro et al., 2017.

métalliques de très grand diamètre $D$ compris entre 4 et $6 \mathrm{~m}$, et pouvant atteindre $8 \mathrm{~m}$ dans les applications les plus récentes (Fig. 2). Leur longueur $L$ est déterminée par leur raideur sous chargement transversal. Leur élancement $L / D$ est limité et généralement inférieur à 10 (Negro et al., 2017). Le comportement de ces pieux, tant en réponse axiale que transversale, est de type rigide contrairement à la plupart des 


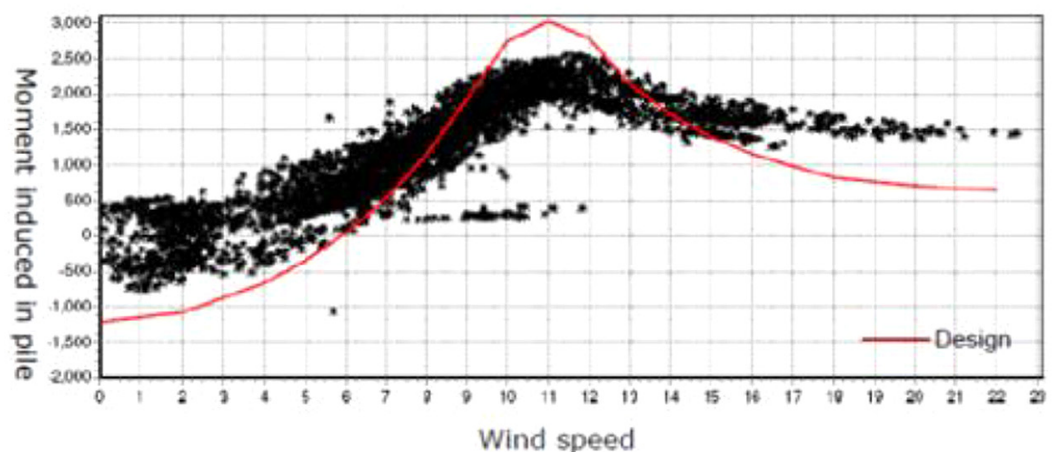

(a)

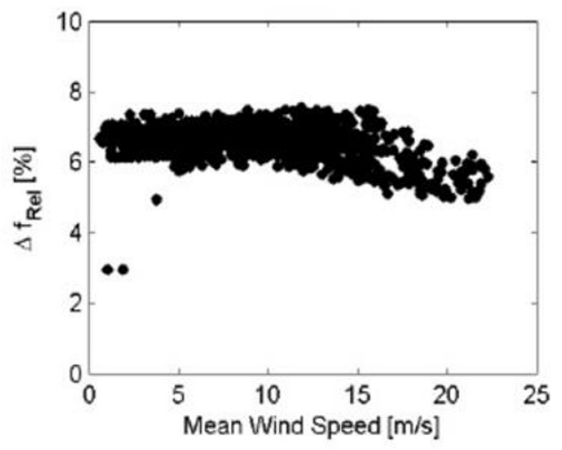

(b)

Fig. 3. Comparaison entre les réponses mesurées et calculées d'un monopieu. a : Moments (Hald et al., 2009); b : Fréquence (Kallehave et al., 2012).

Fig. 3. Comparison between measured and calculated response of a monopile. a: Moments (Hald et al., 2009); b: Frequency (Kallehave et al., 2012).

pieux supportant des structures offshore (jackets) ou de génie civil (bâtiments, ponts, etc.).

L'industrie éolienne s'est initialement appuyée sur le savoir-faire de l'industrie pétrolière (API/ISO), puis l'a progressivement adapté en créant des codes dédiés à l'éolien offshore comme le GL (2005), le DNV-OS-J101 (2014) et le BSH $(2007,2011)$. La méthode classique considérée dans ces guides pour représenter la structure est celle d'une poutre Euler-Bernoulli. À partir du modèle de Winkler, l'interaction sol-structure est représentée par un ensemble de ressorts découplés répartis le long du fût. Cette méthode est classiquement appelée méthode des courbes $p-y$. Cependant, un certain nombre de limitations ou de divergences sont notables dans le cas de l'application au monopieu. En particulier, il est clairement spécifié dans la version 2014 de la norme DNV-OS-J101, que les courbes $p-y$ utilisées jusqu'à présent ne sont pas adaptées au calcul des monopieux rigides de grand diamètre (DNV-OS-J101, 2014 ; Byrne et al., 2015a).

Un aspect très critique du dimensionnement des fondations concerne l'évaluation de la fréquence propre des structures monopodes (ensemble tour + fondation) afin d'éviter tout phénomène de résonance et de limiter la fatigue de la structure. Cette fréquence propre doit rester éloignée à la fois des fréquences de rotation du rotor et des pales (et de leurs premiers harmoniques) et de la fréquence de la houle. La rigidité en rotation de la fondation joue un rôle déterminant dans la réponse globale du système (Kallehave et al., 2015). Elle doit être déterminée pour de faibles valeurs de distorsion $\mathrm{du}$ sol. Les courbes $p-y$ traditionnelles ne sont pas représentatives pour estimer les déplacements transversaux à faible distorsion. Leur utilisation a conduit à fortement sousestimer la raideur en rotation de monopieux installés dans des sables denses, comme cela a été mis en évidence par des mesures de moments et de fréquences réalisées sur des structures installées (Fig. 3).

Par ailleurs, les fabricants de turbines imposent des tolérances très sévères sur l'inclinaison à long terme des axes de rotor. Selon le guide DNV, la rotation globale de la structure est limitée à $0,5^{\circ}\left(0,25^{\circ}\right.$ lors de l'installation et $0,25^{\circ}$ lors de la durée de vie). Le respect de ces exigences suppose une évaluation rigoureuse et précise les déplacements transversaux cumulés générés par le très grand nombre de cycles, et nécessite donc de disposer de méthodes d'ingénierie précises pour évaluer la raideur des monopieux à de faibles niveaux de distorsion et dans tous types de sols.

Les progrès effectués dans le développement de modèles explicites de calcul par la méthode des éléments finis (Wichtmann, 2005; Papon et al., 2012; Burlon et al., 2014) permettent d'espérer à moyen terme le traitement de grands nombres de cycles. Les approches globales (Peralta, 2010; Khemakhem et al., 2012) sont beaucoup plus pertinentes au stade actuel. Il s'agit d'exprimer le déplacement de la tête du pieu $y_{N}$ (ou la rotation du pieu $\theta_{N}$ ) au cycle $N$ en fonction de son déplacement $y_{1}$ (ou rotation $\theta_{1}$ ) au premier cycle. Les expressions proposées dans la littérature sont principalement calées sur des essais de laboratoire sur sables à $1 \times \mathrm{g}$ (LeBlanc et al., 2010; Peralta, 2010) et sur des essais en macrogravité (centrifugeuse) sur sable (Rakotonindriana, 2009; Rosquoët et al., 2013) et sur argiles (Khemakhem et al., 2012). Une comparaison avec des résultats sur pieux in situ a été effectuée dans le cadre du projet SOLCYP+. Ces essais mettent en lumière l'effet de la rigidité du pieu et du mode de mise en place. On peut noter également, depuis quelques années, un nombre important d'études expérimentales sur le comportement cyclique de monopieux (Kirkwood et Haigh, 2014; Yu et al., 2015; Bayton et Black, 2016; Albiker et al., 2017; Abadie et al., 2017, 2018) qui enrichissent la base de données existante pour mieux appréhender le comportement des monopieux.

\section{Particularité des sols français}

Le second point délicat concerne la spécificité des sols français. Les informations géologiques disponibles, confirmées par les reconnaissances géotechniques et géophysiques déjà effectuées sur les sites des futurs champs éoliens, indiquent que les terrains sont principalement constitués, en totalité ou en alternance :

- de sables;

- d'argiles présentant des degrés de consolidation variables;

- de craies; 
- de marnes ;

- de matériaux carbonatés spécifiques allant du sable faiblement cimenté à des calcarénites, assimilables à des roches moyennement dures.

À ce jour, les éoliennes offshore ont été installées majoritairement dans les deux premières catégories de sols.

Le cas des matériaux carbonatés (marnes, craies, calcaires) est complètement absent des normes de l'industrie offshore. L'objectif du projet est ici de capitaliser l'expérience française acquise à terre et en mer dans ce type de sols et de traiter de certains aspects spécifiques, comme la dégradation et le vieillissement de ces terrains sous sollicitations cycliques. À ce jour, dans la plupart des cas, le dimensionnement sous chargement transversal de pieux installés dans des roches carbonatées (massives ou stratifiées) se fait par intégration de courbes de transfert $p-y$ comme pour les sols. Les principales méthodes utilisées dans l'industrie sont celles de Abbs (1983), Fragio et al. (1985), Reese Lymon (1997), Dyson et Randolph (2001) et Erbrich (2004). Ces méthodes ne permettent pas de prendre en compte le comportement de pieux rigides et l'effet des cycles.

Pour les terrains sableux, la difficulté provient de l'absence de recommandations sur l'effet du mode d'installation par battage et sur l'évolution au cours de la vie de l'ouvrage (succession de chargements opérationnels et de tempêtes) des paramètres influant sur l'interaction sol-structure.

\section{Positionnement du projet}

Le projet SOLCYP + s'inscrit dans la continuité des projets français et européens qui se sont terminés récemment: SOLCYP+ et CHARGEOL sur l'effet des cycles sur les pieux flexibles, et PISA sur l'amélioration des connaissances de la capacité latérale statique de monopieux.

Le projet SOLCYP+ s'est attaché à améliorer les connaissances sur le comportement des pieux soumis à des sollicitations cycliques axiales et transversales et à proposer des procédures pour la prise en compte de l'effet des cycles dans le dimensionnement des ouvrages terrestres ou maritimes. Ce projet a permis d'aboutir à des recommandations (Puech et Garnier, 2017), dont les avancées principales concernent les points suivants: caractérisation et traitement des charges cycliques, proposition de critères de sévérité du chargement cyclique, établissement de diagrammes de stabilité cyclique pour différents modes d'installation et de sols, procédures de détermination des lois de dégradation cyclique, développement d'un logiciel de simulation de l'effet des chargements cycliques axiaux (TZC).

Le projet CHARGEOL, sur l'étude du comportement des pieux sous sollicitations cycliques, était consacré aux fondations des éoliennes marines de type jacket sous chargements complexes combinés, monotones et cycliques (Isorna et al., 2015, 2017).

Le projet PISA (Pile Soil Analysis, Byrne et al., 2015a), coordonné par la société Orsted (anciennement DONG), avait pour objectif le développement d'une nouvelle approche pour le dimensionnement des monopieux sous chargement transversal. Il a été divisé en deux phases: une première phase expérimentale concernant des essais sur pieux tests, dans les sables denses (Dunkerque, France) et dans les argiles surconsolidées (Cowden, Royaume-Uni), et une seconde phase d'interprétation. Il est important de noter que l'objectif final est de reproduire le chargement subi sur site par un monopieu de 5 à $10 \mathrm{~m}$ de diamètre installé par battage. Pour des raisons pratiques, le diamètre a été limitée à $2 \mathrm{~m}$ (Byrne et al., 2015b). Les résultats obtenus ont permis une avancée majeure dans la détermination numérique et analytique de la capacité portante des monopieux dans les argiles et les sables. (Byrne et al., 2015a). L'interprétation des données a été limitée aux chargements statiques. Les partenaires du projet sont libres de poursuivre pour leur compte l'interprétation des essais cycliques sous réserve de respecter les clauses de confidentialité.

\section{Objectifs de SOLCYP+}

Les objectifs détaillés de SOLCYP+ consistent à :

- identifier les effets du mode de mise en place des monopieux dans des terrains sableux ou carbonatés sur l'état initial de la fondation et ses conséquences pour le comportement en fonctionnement;

- améliorer les connaissances et les bases de données relatives aux matériaux carbonatés;

- concrétiser les connaissances théoriques et pratiques obtenues dans des projets de recherche récents (SOLCYP+, PISA) et au travers d'essais de pieux réalisés en France par le passé (cas des craies);

- développer des outils de dimensionnement dédiés à l'ingénierie géotechnique marine.

Les méthodes de dimensionnement et de conception doivent permettre de concevoir des systèmes de fondations plus fiables et plus robustes dans un objectif de diminution des risques. À terme, une réduction éventuelle des coûts de conception et de construction peut être envisagée grâce à une approche mieux maîtrisée des interactions entre le sol et les monopieux tout au long de l'exploitation de l'ouvrage.

\section{Le programme scientifique}

Le projet SOLCYP+ a été découpé en en 6 lots :

- lot 1 -Pilotage du projet;

- lot 2-Effets du chargement cyclique sur la réponse transversale des monopieux dans les sables;

- lot 3-Étude du comportement des matériaux carbonatés;

- lot 4-Étude du comportement en vraie grandeur et modélisation des monopieux dans le calcaire;

- lot 5-Méthodes de dimensionnement;

- lot 6-Dissémination, publication et organisation d'événements.

On détaille ci-après uniquement les lots scientifiques 2 à 5 .

\subsection{Lot 2-Effets du chargement cyclique sur la réponse transversale des monopieux dans les sables}

L'objectif est d'étudier en centrifugeuse le comportement des monopieux installés par battage dans les sables denses et chargés transversalement. Comme on l'a indiqué dans la partie contexte, l'effet du mode d'installation est un facteur mal 

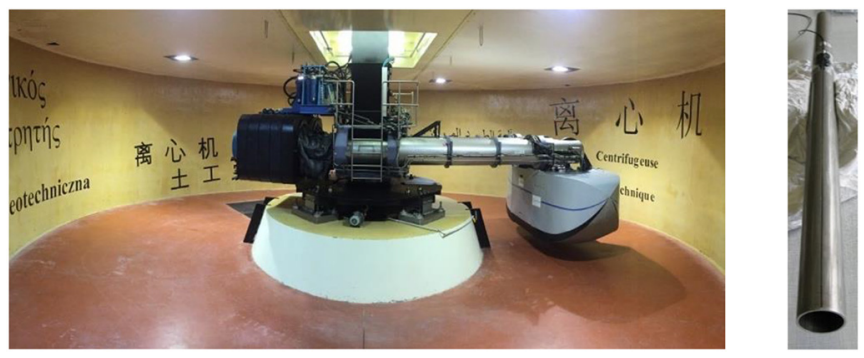

Fig. 4. Centrifugeuse géotechnique de l'IFSTTAR (a) et modèle réduit de monopieu $D=50 \mathrm{~mm}$ avec fibre optique (b).

Fig. 4. Geotechnical centrifuge at IFSTTAR (a) and monopile model $D=50 \mathrm{~mm}$ with optical fiber (b).

connu, en particulier lors de chargement cyclique transversal. Les essais en centrifugeuse permettent d'étudier la réponse globale d'un monopieu sous chargement cyclique transversal et également sous chargement monotone jusqu'à la rupture.

Le dispositif d'essai est la centrifugeuse de l'IFSTTAR de Nantes (Fig. 4); le prototype est un monopieu de $10 \mathrm{~m}$ de diamètre. Les paramètres étudiés sont: l'élancement $L / D$, l'excentrement du point d'application de l'effort $(L e / D)$, le mode de mise en place, le taux de chargement cyclique $\left(H_{\max } /\right.$ $\left.H_{\text {ref }}\right)$. La mise en place d'une instrumentation par fibre optique permet d'avoir des mesures fines des moments de flexion, d'obtenir les pressions et déplacements le long du fût, et de dériver les courbes $p-y$ (Fig. 5).

Deux pieux modèles seront testés : $D=5 \mathrm{~cm}$ et $D=10 \mathrm{~cm}$. Le second permet d'avoir une meilleure définition de la distribution des moments le long du fût, en particulier près de la pointe.

Un dispositif de battage en vol qui permettra d'étudier l'effet du mode de mise en place est en cours de développement à l'IFSTTAR. Ce dispositif sera mis au point et testé dans le cadre du projet.

L'ensemble des résultats obtenus sera utilisé dans le lot 5 pour la validation des modèles numériques.

\subsection{Lot 3-Étude du comportement des matériaux carbonatés}

Dans ce lot, l'objectif est d'étudier les propriétés mécaniques et géométriques de l'interface sol-coulis de scellement dans les roches tendres carbonatées. Dans ce type de terrain, le monopieu est foré et scellé dans la roche encaissante par un coulis approprié. L'objectif final est d'obtenir des lois d'interface intégrables à des fins de dimensionnement.

Le dispositif utilisé est la machine BRC3D(boîte de cisaillement pour roche avec chargement selon 3 directions) du laboratoire 3SR (Fig. 6) qui est bien adaptée à la détermination de ces lois locales (Boulon, 1995; Tata et al., 2015).

La roche retenue pour cette étude est une calcarénite du site de Gouvieux, utilisée dans le lot 4 pour les essais en vraie grandeur.

Le programme d'essais comporte à la fois des essais de caractérisation des matériaux (roche, coulis), des essais de cisaillement monotone (roche-coulis, roche-roche et couliscoulis), des essais cycliques de cisaillement d'interface (configurations identiques aux essais monotones), en se

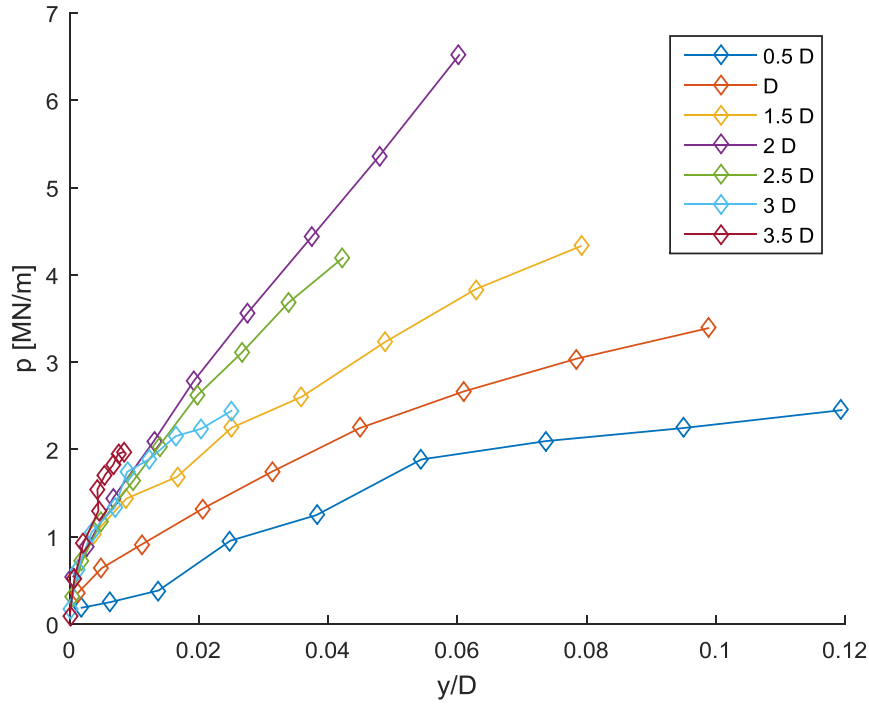

Fig. 5. Exemples de courbes $p-y$ obtenues en centrifugeuse pour plusieurs profondeurs.

Fig. 5. Examples of $p-y$ curves obtained in centrifuge for several depths.

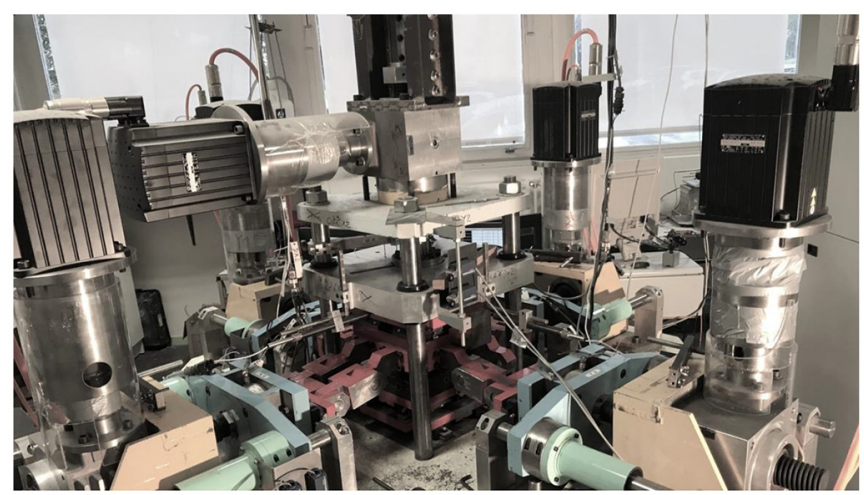

Fig. 6. Le dispositif BCR3D.

Fig. 6. The BCR $3 D$ apparatus.

rapprochant autant que possible des conditions de terrain en termes de saturation, de niveaux de contrainte normale effective et de rugosité de l'interface. Les conditions utilisées pour ces essais sont dites CNL (Constant Normal Load) et CNS (Constant Normal Stiffness). Cette dernière a été privilégiée pour les essais, car plus représentative du comportement de l'interface sol-pieu.

Un exemple de résultats obtenus lors d'un essai cyclique en condition CNS sur l'interface roche carbonatée-coulis est présenté sur la figure 7. Cet essai permet de définir le nombre de cycles à la rupture que l'on reporte dans un diagramme d'interaction (Puech et Garnier, 2017).

\subsection{Lot 4-Étude du comportement en vraie grandeur et modélisation des monopieux dans le calcaire}

Dans le cadre du développement des projets éoliens en mer de St Nazaire et Courseulles, le groupement EMF (EDF EN et 

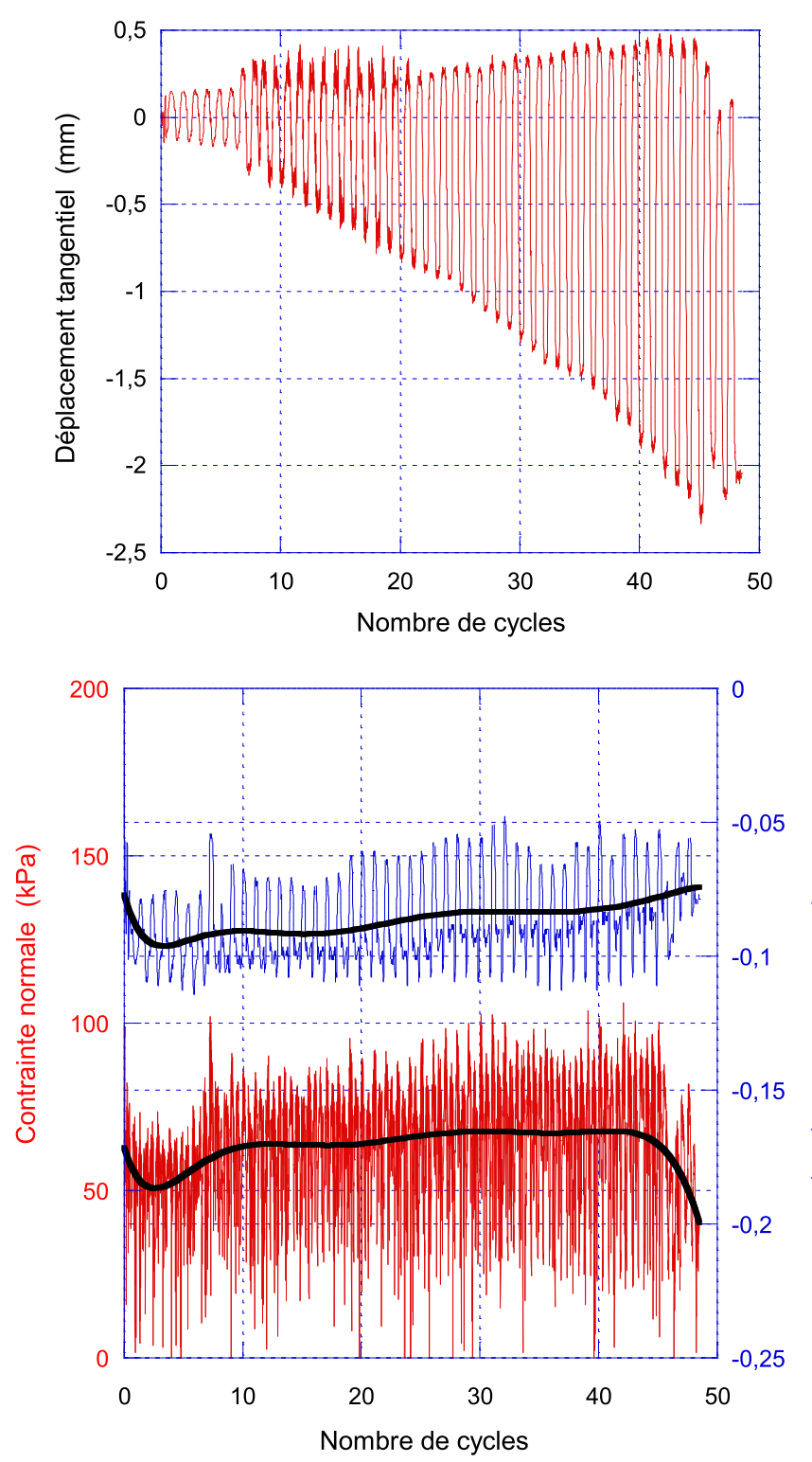

Fig. 7. Résultats de l'essai cyclique $\mathrm{H} 3$ alterné roche/coulis en condition CNS $\left(k_{n}=1500 \mathrm{kPa} / \mathrm{mm}, \sigma_{n 0}=100 \mathrm{kPa}, \tau_{\text {moy }}=0 \mathrm{kPa}\right.$, $\tau_{\text {cyc }}=77,5 \mathrm{kPa}$ ).

Fig. 7. Results of alternated cyclic test $\mathrm{H3}$ rock/grout in CNS condition $\left(k_{n}=1500 \mathrm{kPa} / \mathrm{mm}, \sigma_{n 0}=100 \mathrm{kPa}, \tau_{\text {moy }}=0 \mathrm{kPa}, \tau_{c y c}=\right.$ $77.5 \mathrm{kPa}$ ).

Enbridge) et WPD a lancé une campagne de pieux tests dans des calcarénites (11 pieux dont 9 chargés transversalement) en 2016 (Fig. 8). Cette campagne, inspirée du programme de recherche PISA, a comporté à la fois des essais monotones et cycliques et une instrumentation importante des pieux tests. Afin de capitaliser les connaissances sur ces essais de pieux, EDF EN et EDF R\&D ont lancé une thèse relative à l'interprétation des essais cycliques conjointement avec l'École des Ponts ParisTech (Navier-Géotechnique) et l'Université de Western Australia (UWA). L'objectif est de développer une méthodologie d'évaluation de l'interaction solstructure dans les calcaires sous chargement cyclique

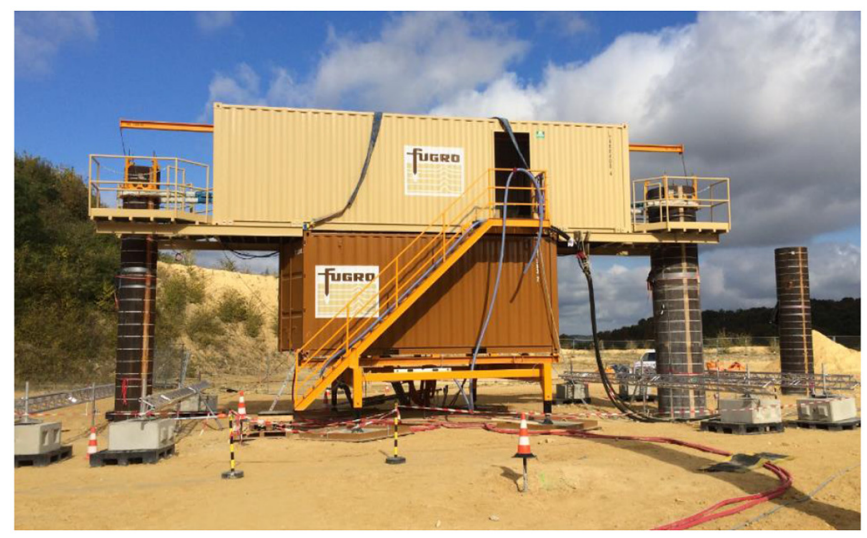

Fig. 8. Vue du dispositif de chargement pour les essais en vraie grandeur (EDF-EN, site de Gouvieux, Oise, France).

Fig. 8. View of the loading device for full-scale tests (EDF-EN, Gouvieux site, Oise, France).

transversal. Ce travail se décompose en plusieurs phases: une première consiste à caractériser le comportement du calcaire sous chargement cyclique, afin d'orienter et de calibrer les modèles développés dans la phase 2 . La seconde phase consiste à développer un modèle analytique en s'appuyant dans une première étape sur le modèle CHIPPER (Erbrich, 2004). Finalement, ces travaux doivent conduire à la proposition d'une méthodologie de dimensionnement robuste et fiable vis-à-vis de scénarios de défaillance (fissuration dans la roche, zone de contact), et donc une meilleure calibration des courbes $p-y$. Ce lot n'étant pas financé par le projet SOLCYP+, les résultats sont confidentiels, à l'exception de la caractérisation de la roche (physique et mécanique) qui sera réalisée au laboratoire Navier de l'École des Ponts ParisTech.

\subsection{Lot 5-Méthodes de dimensionnement}

L'objectif de ce lot est le dimensionnement des pieux et des monopieux soumis à des charges cycliques, en s'appuyant sur des outils de calcul fiables et robustes qui doivent permettre la prise en compte de phénomènes complexes. Les deux principaux objectifs sont: le développement d'outils de calculs qui permettent l'estimation, en fonction des cycles de chargement, des déplacements et des efforts dans les monopieux. À partir des essais en centrifugeuse (lot 2) et des bases d'essais de laboratoire complétées pour partie dans le lot 3, il s'agit d'identifier, à l'échelle locale, les phénomènes associés à l'accroissement des déformations et des déplacements avec les cycles. Les bases de données à différentes échelles notamment à partir des essais dans le lot 2 , doivent permettre la validation des outils de calculs développés.

Une première tâche consiste à finaliser le développement de la méthode de dimensionnement des pieux sous charge cyclique axiale. Ces travaux initiés dans le cadre du projet SOLCYP+, avec le développement d'une méthode de calcul de type $t$ - $z$ cycle à cycle (Burlon et al., 2014; Abchir et Burlon, 2018), doivent être complétés par des comparaisons avec d'autres résultats d'essais in situ et finalement aboutir à une 

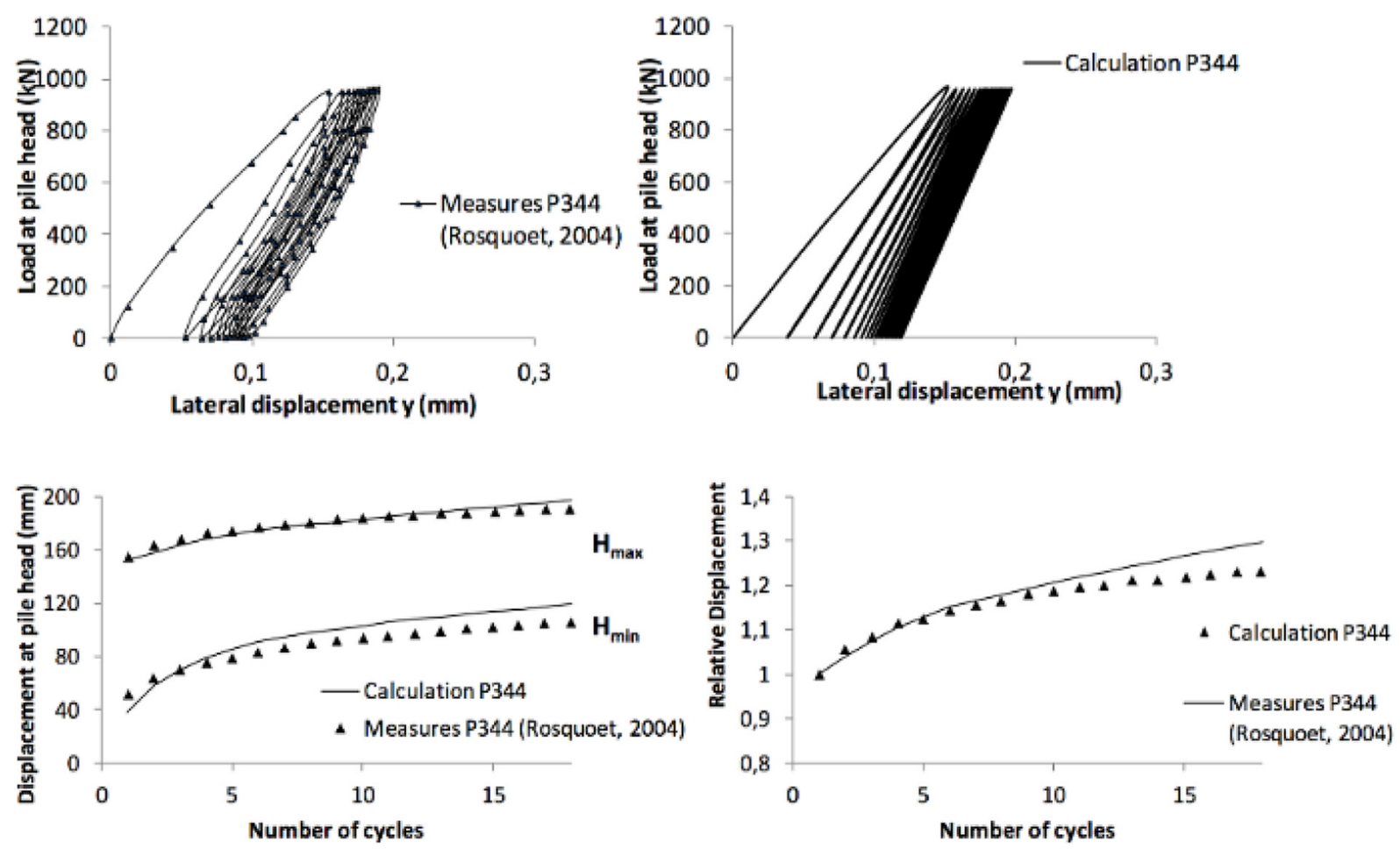

Fig. 9. Comparaison entre résultats numérique (PILATE CYC) et expérimental (essais en centrifugeuse, Rosquoët, 2004) (Abchir et Burlon, 2018). Fig. 9. Comparison between numerical results (PILATE CYC) and experimental results (centrifuge tests, Rosquö̈t, 2004) (Abchir and Burlon, 2018).

méthode complète prête à être utilisée par les ingénieurs ainsi qu'au développement d'un logiciel (Abchir et Burlon, 2018).

La seconde tâche consiste à proposer une loi de dégradation de la résistance des pieux sous charge cyclique transversale. Comme pour le cas des charges axiales, on s'appuiera sur les lois développées dans le cadre du projet SOLCYP + , en particulier la méthode globale (déplacement et moment après une séquence donnée) et la méthode locale (courbes $p-y$ et coefficients d'abattement). Une première étape consiste à comparer ces approches à des résultats d'essai de pieux en vraie grandeur (projetPISA) et d'essais en centrifugeuse (lot 2) afin de valider les méthodes développées et finalement de proposer un nouveau modèle $p-y$ réalisant un calcul cycle à cycle et prenant en compte l'effet des cycles sur le comportement du pieu et l'interaction sol-pieu (Fig. 9). Ces courbes seront ensuite implémentées dans le logiciel PILATE de l'IFSTTAR.

Une seconde tâche consiste à développer des modèles numériques et des outils de calcul destinés à reproduire le comportement axial des pieux dans la craie (Fig. 10). Ce travail sera réalisé dans le cadre d'une thèse au LGCgE. Il s'agit d'identifier les mécanismes majeurs à prendre en compte dans les modèles, puis de considérer divers types de chargements. De nombreux travaux consacrés au comportement mécanique de la craie existent déjà: au LGCgE avec des essais en laboratoire et de nombreuses lois de comportement rendant compte des phénomènes de fluage ou de dégradation et à l'IFSTTAR avec de nombreux essais de chargement de pieux dans la craie. La première étape du travail doit aboutir à une

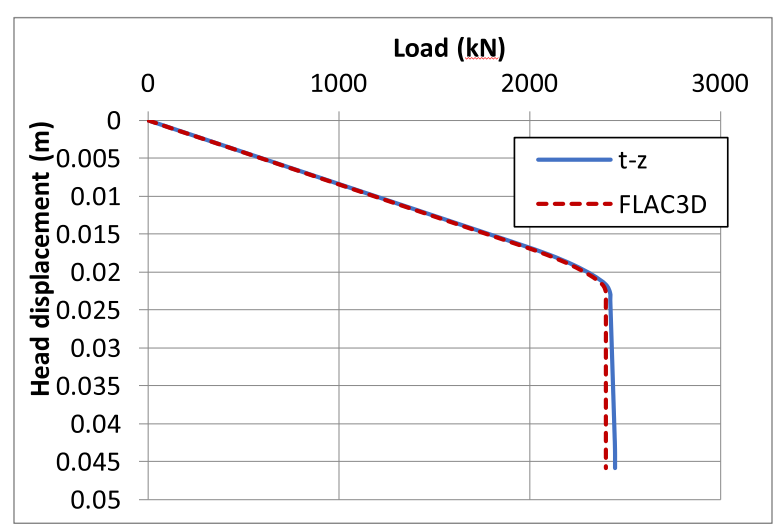

Fig. 10. Comparaison entre modèle hybride craie-interface-pieu et la méthode $t-z$ pour le calcul du déplacement en tête de pieu.

Fig. 10. Comparison between the hybrid model chalk-interface-pile and the $t$-z method for calculating the displacement at the head of the pile.

synthèse du comportement de pieux et de monopieux mis en place dans la craie. La seconde étape doit fournir des recommandations pour l'utilisation de modèles numériques, plutôt à destination de l'ingénierie. Dans un second temps, on souhaite considérer de nouvelles approches dans la modélisation, telles que les macroéléments, qui permettront plus facilement d'intégrer l'évolution de la craie dans les modèles 
numériques, ou plutôt l'évolution de ses propriétés en fonction du niveau et du caractère cyclique du chargement.

Finalement une dernière tâche, pilotée par INNOSEA, a pour objectif de mettre en application les méthodes de dimensionnement de monopieux développées lors des tâches précédentes sur un cas pratique représentatif d'un site français.

\section{Conclusion}

Le développement des éoliennes marines posées sur le fond est un enjeu à la fois industriel et économique pour le secteur des énergies renouvelables. Le projet SOLCYP+ doit permettre de développer de nouvelles méthodologies fiables et robustes pour le dimensionnement des monopieux de grand diamètre. Ce projet s'appuie à la fois sur des essais en laboratoire (centrifugeuse, BRC3D), sur des essais in situ et sur la modélisation. On a, en particulier, mis l'accent sur l'effet du mode de mise en place par battage dans les sables denses, le comportement de l'interface pieu-roche carbonaté dans le cas des pieux forés, et sur le développement d'outils numériques applicables par les bureaux d'ingénierie. Ce projet s'inscrit dans la continuité des projets nationaux (SOLCYP+, CHARGEOL) et européens (PISA, ALPACA). Le consortium regroupe à la fois des compétences académiques et industrielles reconnues dans les domaines des fondations, du comportement des sols et des roches, des modèles physiques et de l'éolien posé en mer.

Remerciements. Ce travail a bénéficié d'une aide de France Energies Marines (FEM) et de l'État, gérée par l'Agence Nationale de la Recherche au titre du programme Investissements d'avenir portant la référence ANR-10-IEED-0006-19. Les auteurs tiennent à remercier l'ensemble des partenaires pour leur soutien financier au projet : EDF EN, EDF R\&D, FUGRO, FEM, IFSTTAR, INNOSEA, ORSTED, École des Ponts ParisTech, Polytech de Lille et Université de Grenoble Alpes.

\section{Références}

Abadie C, Byrne B, Houlsby G. 2017. Modelling of monopile response to cyclic lateral loading in sand. Offshore Site Investigation Geotechnics 8th International Conference Proceedings. Society of Underwater Technology, pp. 1046-1053. DOI: 10.3723/OSIG17.1046.

Abadie CN, Byrne BW, Houlsby GT. 2018. Rigid pile response to cyclic lateral loading: Laboratory tests. Géotechnique 1-14. DOI: 10.1680/jgeot.16.p.325.

Abbs AF. 1983. Lateral pile analysis in weak carbonate rocks. Geotechnical Practice in Offshore Engineering, ASCE, Austin, Texas, pp. 546-556. DOI: 10.1139/cgj-2015-0600.

Abchir Z, Burlon S. 2018. Monopile design methodology under axial cyclic loading - User manual of the axial TZC. SOLCYP+ project, WP5, Delivrable 02.

Albiker J, Achmus M, Frick D, Flind F. 2017. $1 \mathrm{~g}$ model tests on the displacement accumulation of large-diameter piles under cyclic lateral loading. Geotech Test J 40: 20160102. DOI: 10.1520/GTJ20160102. API RP2A-WSD . 2000. Recommended practice for planning, designing, and constructing fixed offshore platforms. API Recommended Practice 2A-WSD, 21st edition, Dallas.
Bayton SM, Black JA. 2016. The effect of soil density on offshore wind turbine monopile. Proc 3rd Eur Conf Phys Model (Eurofuge 2016) 1: 245-251.

Boulon M. A 3-d direct shear device for testing the mechanical behaviour and the hydraulic conductivity of rock joints. In: Proceedings Mechanics of Jointed and Faulted Rock, 1995, pp. 407-413.

BSH. 2007. Standard-Design of offshore wind turbines. Hamburg: Federal Maritime and Hydrographic Agency.

BSH. 2011. Guidance for use of the BSH standard "Design of offshore wind turbines, 2007". Hamburg: Federal Maritime and Hydrographic Agency.

Burlon S, Mroueh H, Cao J. 2014. "Skipped cycles" method for studying cycling loading and soil-structure interface. Comp Geotech 61: 209-220. DOI: 10.1016/j.compgeo.2014.05.007.

Byrne B, McAdam R, Burd H, et al. 2015a. New design methods for large diameter piles under lateral loading for offshore wind applications. In: Frontiers in offshore geotechnics III. DOI: 10.1201/b18442-96.

Byrne B, McAdam R, Burd H, et al. 2015b. Field testing of large diameter piles under lateral loading for offshore wind applications. Proceedings of the 16th European Conference on soil Mechanics and Geotechnical Engineering (ECSMGE), Edinburgh, UK.

Danish Geotechnical Society. 2014. Disponible sur https://danskgeo tekniskforening.dk/sites/default/files/pdf/pdf2014/Moede $\% 202 /$ Session $\% 203 \% 20-\% 20$ Presentation $\% 202 \% 20-\% 20$ A $\% 20$ Muir\% 20Wood\%20-\%20DONG\%20Energy\%20-\%20Future\%20Mono pile\%20Design\%20-\%20DGF\%20Seminar\%202014-04-01.pdf (dernière consult. 2019/11/06).

DNV-OS-J101. 2014. Design of offshore wind turbine structures. Oslo: DNV Offshore Standards, Det Norske Veritas.

Dyson GJ, Randolph MF. 2001. Monotonic lateral loading of piles in Calcareous sediments. J Geotech Eng Div, ASCE 127(4): 346-352. DOI: 10.1061/(ASCE)1090-0241(2001)127:4(346).

Erbrich CT. 2004. A new method for the design of laterally loaded anchor piles in soft rock. Proceedings of Offshore Technology Conference in Houston, Texas, USA, 3-6 May 2004.

Fingersh L, Hand M, Laxson A. 2006. Wind turbine design cost and scaling model. Technical report NREL/TP-500-40566. Golden, Colorado: National Renewable Energy Laboratory.

Fragio AG, Santiago JL, Sutton VJR. 1985. Load tests on Grouted piles in rock. Proceedings Offshore Technology Conference, OTC 4851.

GL. 2005. Rules and Guidelines. IV Industrial Services. Part 2-Guidelines for the certification of offshore wind turbines. Germanischer Lloyd, Reprint 2007.

Hald T, Morch C, Jensen L, Bakmar CL, Ahle K. 2009. Revisiting monopile design using $p-y$ curves. Results from full-scale measurements on Horms Rev. Proceeding of the European Offshore Wind Conference.

Isorna R, Dano C, Kotronis P, Thorel L, Blanc M, Philippe M. 2015. Projet CHARGEOL : étude expérimentale et numérique des pieux de fondation des éoliennes offshore. $12^{e}$ Colloque National en calcul des Structures, Giens, France.

Isorna R, Blanc M, Thorel L, Kotronis P, Dano C, Philippe M. 2017. Axial behaviour of jacket piles for offshore wind turbines. Int $J$ Phys Model Geotech 17: 229-245. DOI: 10.1680/ jphmg.15.00044.

Kallehave D, LeBlanc C, Liingaard MA. 2012. Modification of the API $p-y$ formulation of initial stiffness of sand. Offshore Site Investigation and Geotechnics: Integrated Technologies-Present and Future, London, UK, pp. 465-472. 
Kallehave D, Byrne B, LeBlanc C, Mikkelsen K. 2015. Optimization of monopiles for offshore wind turbine. Philos Trans Royal Societ A 373. DOI: 10.1098/rsta.2014.0100.

Khemakhem M, Chenaf N, Garnier J, Favraud C, Gaudicheau P. 2012. Development of degradation laws for describing the cyclic lateral response of piles in clays. 7th International Conference Offshore Site Investigation and Geotechnics, SUT, London.

Kirkwood P, Haigh S. 2014. Centrifuge testing of monopiles subject to cyclic lateral loading. In: ICPMG2014-Physical Modelling in Geotechnics. CRC Press, pp. 827-831. DOI: 10.1201/b16200-114.

LeBlanc C, Houlsby GT, Byrne BW. 2010. Response of stiff piles in sand to long-term cyclic lateral loading. Géotechnique 60: 79-90. DOI: $10.1680 /$ geot.7.00196.

Ministère de la Transition écologique et solidaire. 2019. Disponible sur https:/www.ecologique-solidaire.gouv.fr/sites/default/files/ Synthèse $\% 20$ finale $\% 20$ Projet $\% 20$ de $\% 20$ PPE.pdf (dernière consult. 2019/12/06).

Negro V, López-Gutiérrez J-S, Esteban MD, Alberdi P, Imaz M, Serraclara J-M. 2017. Monopiles in offshore wind: Preliminary estimate of main dimensions. Ocean Eng 133: 253-261. DOI: 10.1016/j.oceaneng.2017.02.011.

Papon A, Yin Z-Y, Riou Y, Hicher P-Y. 2012. Time homogenization for clays subjected to large numbers of cycles. Int J Numer Anal Method Geomech 37(11): pp. 1470-1491. DOI: 10.1002/nag.2092.

Peralta PK. 2010. Investigations on the behavior of large diameter piles under long-term lateral cyclic loading in cohesionless soil. Degree of Doctor of Engineering, Leibniz University Hannover, $223 \mathrm{p}$.
Puech A, Garnier J. 2017. Recommandations pour le dimensionnement des pieux sous chargements cycliques. Projet National SOLCYP, Editions ISTE.

Rakotonindriana J. 2009. Comportement des pieux et des groupes de pieux sous chargement latéral cycliques. Thèse ENPC. Disponible sur https://pastel.archives-ouvertes.fr/pastel-00575332/document.

Reese Lymon C. 1997. Analysis of laterally loaded piles in weak rock. J Geotech Environ Eng. DOI: 10.1061/(ASCE)1090-0241(1997) 123:11(1010).

Rosquoët F. 2004. Pieux sous chargement latéral cyclique. Thèse de Doctorat, École centrale et Université de Nantes.

Rosquoët F, Thorel L, Garnier J, Chenaf N. 2013. Pieu sous charge latérale dans les sables : développement de lois de dégradation pour prendre en compte l'effet des cycles. Proceedings 18th ICSMGE, Paris.

Tata B, Boulon M, Puech A. 2015. Essais de cisaillement cyclique sur des interfaces calcarénite-coulis. Rapport du Projet National SOLCYP.

Velarde J, Bachynski EE. 2017. Design and fatigue analysis of monopile foundations to support the DTU $10 \mathrm{MW}$ offshore wind turbine. Energy Proc 137: 3-13. DOI: 0.1016/j.egypro.2017.10.330.

Wichtmann T. 2005. Explicit accumulation model for non-cohesive soils under cyclic loading. In: Triantafyllidis T, ed. Schriftenreihe des Institutes für Grundbau und Bodenmechanik der Ruhr, Universität Bochum, Heft 38.

Yu LQ, Wang LZ, Guo Z, et al. 2015. Long-term dynamic behavior of monopile supported offshore wind turbines in sand. Theor Appl Mech Lett 5: 80-84. DOI: 10.1016/j.taml.2015.02.003.

Citation de l'article : Jean-Claude Dupla, Elisabeth Palix, Guillaume Damblans, Alain Puech, Mathieu Blanc, Christophe Dano, Hussein Mroueh, Marc Kham, Yiorgos Perikleous, Yves Marin, Sebastien Burlon. Le projet ANR SOLCYP+ pour améliorer le dimensionnement des monopieux utilisés comme fondations d'éoliennes marines. Rev. Fr. Geotech. 2019, 158, 4. 